\title{
Präwürmzeitliche Eiskeile und Kryoturbationsformen östlich Bordeaux - eine Mitteilung zur Ausdehnung des vorzeitlichen Permafrostbereichs in Südwestfrankreich
}

\author{
KARL-UlRICH BROSCHE *)
}

\author{
Terraces, sand, gravel, shingle, cryoturbation, \\ ice wedges, interpretation, permafrost, spatial distribution \\ Dordogne, Aquitaine Basin
}

\begin{abstract}
Aus dem Südwesten Frankreichs liegen bisher sehr wenige Belege für die ehemalige Existenz von Permafrost vor. So wird in der Karte von KAISER (1960, Tafel 1), die in etwas erweiterter Form bei WASHBURN (1979, S. 296) abgedruckt ist, nur ein würmzeitliches Vorkommen von Eiskeilen aus dem Gebiet westlich von Bordeaux angegeben, das wahrscheinlich auf den Untersuchungen von BASTIN \& CAILLEUX (1941) beruht. Die nächsten weiter nördlich gelegenen Vorkommen von Eiskeilen befinden sich bei Angoulème an der Charente. Grundlage für diese Teile der Karte von KAISER (1960) ist die Karte von TRICART in CAILLEUX, GuILCHER \& TriCART (1956, Plate III, 34).
\end{abstract}

Das hier mitzuteilende und abzubildende Vorkommen wurde im Sommer 1987 am südlichen Talhang des Isle-Tales $100 \mathrm{~m}$ östlich des Ortsschildes von Mussidan an der Nationalstraße N89 Libourne-Perigeux gefunden. In der „Sablière du Cabillon” wurde, heute von einem Castrol-Michelin-Dienst genutzt, deutlich $(7-10 \mathrm{~m})$ oberhalb der Niederterrasse eine Wechselfolge von hellen, z. T. rostigen Sanden, lehmigen Sanden, Kiesen und Schottern angetroffen, die eine 20-30 m hohe Terrasse aufbauen. Die nach W exponierte Wand ist in halber Höhe gut aufgeschlossen und läßt einerseits kryoturbate Schichtenstörungen intensiver Art in einem ca. $1 \mathrm{~m}$ mächtigen Horizont (Abb. 1), andererseits mehrere fossile Eiskeile erkennen, von denen ein Exemplar in Abb. 2 erkennbar ist. Ein hangendes kiesig-sandiges Sediment stellt hier die Füllmasse für einen oben $30-40 \mathrm{~cm}$ breiten und über 1,2 m langen Eiskeil dar. Auch in Abb. 1 ist

\footnotetext{
*) Anschrift des Verfassers: Prof. Dr. K.-U. BrosChe, Institut für Physische Geographie der FU Berlin, 1 Berlin 41, Grunewaldstr. 35.
}

etwas rechts oberhalb des Kopfes des rechten Kindes ein fossiler Eiskeil zu erkennen, dessen Länge aber nur bei 0,8 bis $1,0 \mathrm{~m}$ liegt. Das Eiskeilfüllmaterial ist rötlich-bräunlich verwittert, wie es in Mitteleuropa typisch für das Füllmaterial saalezeitlicher und älterer Eiskeile in vergleichbaren Sedimenten ist. Die Kombination von intensiven Kryoturbationserscheinungen und von fossilen Eiskeilen läßt keinen Zweifel an ehemaligen Permafrostbedingungen aufkommen. Dabei bleibt offen, um welche Art von Permafrostboden es sich gehandelt hat. Die Eiskeile und Kryoturbationsformen sind als syngenetische Formen i. S. von GALLWITZ (1949) anzusehen und müssen in eine Kaltzeit vor d e r W ü r m a l t z e i t gestellt werden.

Damit ist für diesen Raum ein erster Beleg für einen präwürmzeitlichen Permafrostboden erbracht. Präwürmzeitliche Eiskeile wurden $\mathrm{m}$. W. so weit südwestlich in Frankreich noch nicht festgestellt. Ob die von KAISER (1960, Tafel 1, erweitert in WASHBURN 1979, S. 296) im Südwesten Frankreichs eingezeichnete Grenze des eiszeitlichen kontinuierlichen, möglicherweise auch diskontinuerlichen Permafrostbodens so weit südlich verlief wie auf der Karte (Tafel 1), müssen weitere Untersuchungen zeigen. Denn bisher wurden südlich von Bordeaux und südlich des Eiskeilvorkommens bei Mussidan lediglich Kryoturbationserscheinungen, sortierte fossile Formen von Strukturböden, klimatisch bedingte asymmetrische Täler, Nivationsnischen, grèzes litées (éboulis stratifiés), blockige oder kiesige mudflows, festoons und garlands (festons), niveo-äolische Sande, vertikal orientierte Steine und "periglacial fans" gefunden (vgl. TRICART 1956 in CaIlleuX, Guilcher \& Tricart 1956 zit. nach WASHBURN 1979, S. 294). 

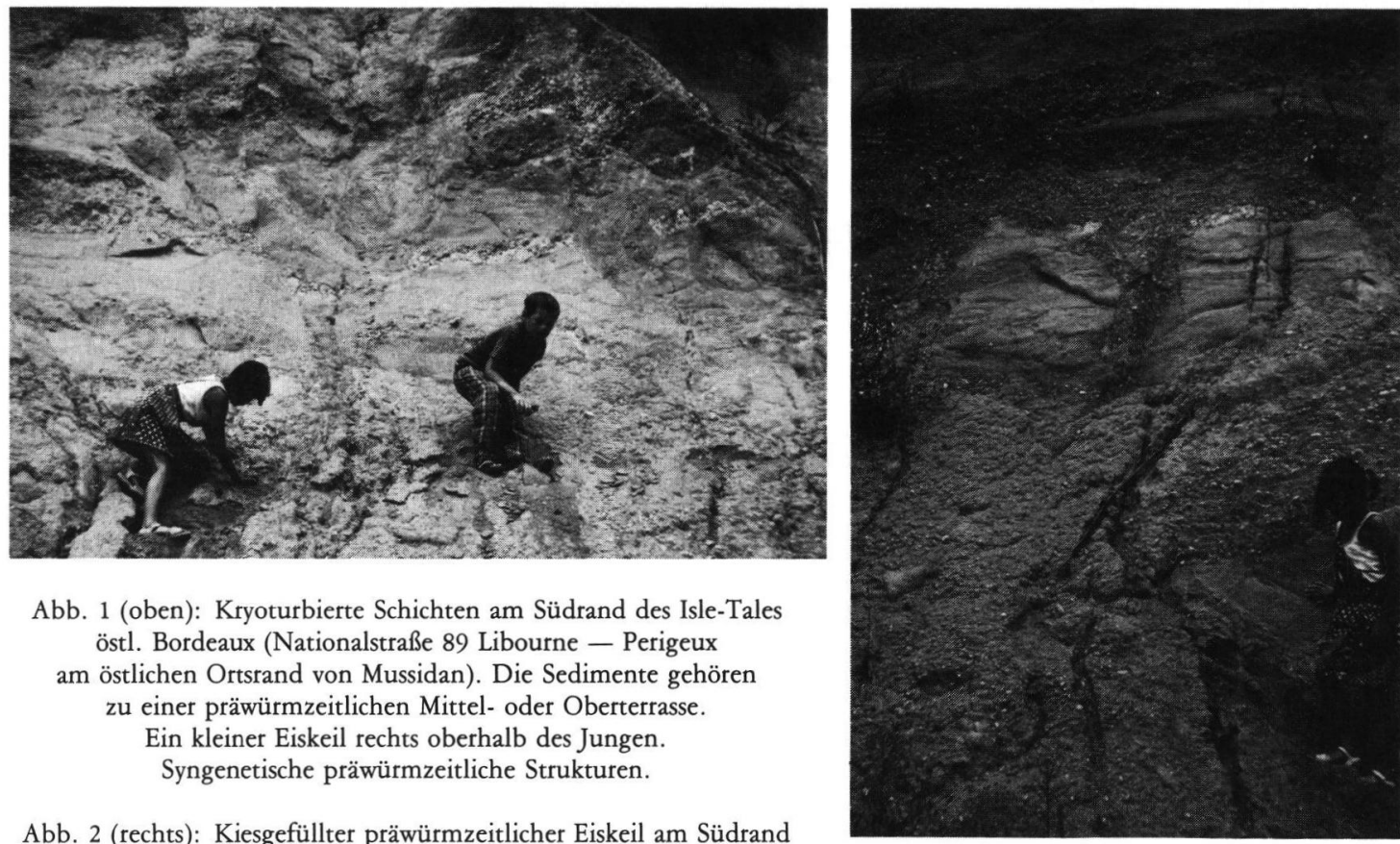

Abb. 1 (oben): Kryoturbierte Schichten am Südrand des Isle-Tales östl. Bordeaux (Nationalstraße 89 Libourne - Perigeux am östlichen Ortsrand von Mussidan). Die Sedimente gehören zu einer präwürmzeitlichen Mittel- oder Oberterrasse.

Ein kleiner Eiskeil rechts oberhalb des Jungen.

Syngenetische präwürmzeitliche Strukturen.

Abb. 2 (rechts): Kiesgefüllter präwürmzeitlicher Eiskeil am Südrand des Isle-Tales östl. Bordeaux im Zentrum des Bildes

(gleiche Lokalität wie bei Abb. 1). Präwürmzeitliche syngenetische Form in einer Mittel- oder Oberterrasse.

Länge des Eiskeils 1-1,2 m.

\section{Schriftenverzeichnis}

Bastin, A. \& Cailleux, A. (1941): Action du vent et du gel dans la région bordelaise. Bulletin de la Societé Géologique de France.

GaLlWITZ, H. (1949): Eiskeile und glaziale Sedimentation. - Geologica 2 (1949), S. 1-24; Berlin.

KAISER, K. (1960): Klimazeugen des periglazialen Dauerfrostbodens in Mittel- und Westeuropa. - Eiszeitalter und Gegenwart 11 (1960), S. 121-141; Öhringen/ Württ.
Cailleux, A., Guilcher, A. \& Tricart, J. (1956): Phénomènes périglaciaires d'age présumé Würm: 34, Plate 3 in Tricart, Jean, Cartes des phénomènes périglaciaires quaternaires en France: Carte géologique detaillé de la France (Mémoires) $40 \mathrm{~S}$.

Washburn, A. L. (1970): Cryopedology. Edward Arnold. 406 S., London.

Manuskript eingegangen am 7.1. 1988,

Nachträge Mai 1989. 